\title{
Bioinformation
}

\section{SPCalc: A web-based calculator for sample size and power calculations in micro-array studies}

\author{
Weiliang Qiu ${ }^{1 *}$ and Mei-Ling Ting Lee ${ }^{2}$ \\ ${ }^{1}$ Channing Laboratory, Brigham and Women's Hospital, Harvard Medical School, 181 Longwood Avenue, Boston, MA 02115; \\ ${ }^{2}$ Division of Biostatistics, School of Public Health, The Ohio State University, Room B-122, Starling-Loving Hall, 320 W. 10th \\ Avenue, Columbus, OH 43210-1240; \\ Weiliang Qiu* - Email: stwxq@channing.harvard.edu;* Corresponding author \\ received November 13, 2006; accepted November 21, 2006; published online November 22, 2006
}

\begin{abstract}
:
Calculation of the appropriate sample size in planning microarray studies is important because sample collection can be expensive and time-consuming. Sample-size calculation is also a challenging issue for microarray studies because the number of genes is far larger than the number of samples so that traditional methods of sample-size calculation cannot be directly applied. To help investigators answer the question of how many samples are needed in their microarray studies, we developed a user-friendly web-based calculator, SPCalc, for calculating sample size and power for a variety of commonly used experimental designs, including completely randomized treatmentcontrol design, matched-pairs design, multiple-treatment design having an isolated treatment effect, and randomized block design.
\end{abstract}

Availability: The web-based calculator SPCalc is publicly available at http://www.biostat.harvard.edu/people/faculty/mltlee/webfront-r.html

Keywords: gene expression; microarray; sample size; power calculation

\section{Background:}

It is often expensive and time consuming to obtain biological samples for microarray studies. To obtain statistical power for testing whether genes are differentially expressed across experimental conditions, investigators need to know the minimal sample size required for their experiments. On the other hand, if microarray experiments are done with available samples only, investigators need to know the statistical power of the test.

We developed a web-based calculator, called SPCalc, to help investigators calculate (1) sample size and (2) power in the planning stage of a microarray study. This program helps investigators to determine how many samples are needed to achieve a specified power for testing differentially expressed genes. Conversely, this program can help determine the power when the study sample size is given.

To the best of our knowledge, SPCalc is the only web-based calculator for sample-size and power calculation for microarray studies. The web interface of SPCalc is user-friendly. It is simple to use and is well documented.

\section{Methodology:}

This web-based calculator, which is based on methodologies described in [1] and [2], provides two utilities for sample-size calculations for two types of experimental designs (a completely randomized treatment-control design and a matched-pairs design) and three utilities for power calculation for four types of experimental designs (a completely randomized treatmentcontrol design, a matched-pairs design, a multiple-treatment design having an isolated treatment effect, and a randomized block design).

\section{Input}

For both sample-size and power calculation utilities, there are five input text fields corresponding five input parameters. Clicking the Calculate button submits input parameters. A Clear button is used to clear inputs in all five input fields. Validation of the values of input fields is performed after the Calculate button is clicked.

\section{Output}

For sample-size calculation utilities, there are three output text fields corresponding to (1) statistical difference between treatment and control conditions under $\mathrm{H}_{1}$, (2) sample size $\mathrm{n}$ for each group, and (3) total sample size $2 \mathrm{n}$ needed for the study, respectively. For power calculation utilities, there are two output text fields corresponding to (1) non-centrality parameter $\psi_{1}$, and (2) power, respectively.

\section{Caveats and Future Development:}

SPCalc was written via JavaScript and can be run via any JavaScript-enabled web browser. The current version of SPCalc uses the Sidak approach to control type I error. The new version of SPCalc will add options to control false discovery rates. [3]

\section{Acknowledgement:}

This project was support by NIH grant HG02510 (Lee, M-L.T.).

\section{References}

[01] M. L. T. Lee, Analysis of Microarray Gene Expression Data, (2004)

[02] M. L. T. Lee, \& G. A. Whitmore, Statistics in Medicine, 21:3543 (2002) [PMID: 8215016]

[03] Y. Benjamini \& Y. Hochberg, Journal of the Royal Statistical Society, B57:289(1995) 
Sample Size and Power Calculations for Microarray Studies - Dr. Mei-Ling Ting Lee's personal webpage

\section{Sample Size and Power Calculations for Microarray Studies}

This website has been prepared to assist users in computing either a sample size or power value for a microarray experimental study. The user is referred to the cited references for technical background on the methodology underpinning these calculations. This site provides support for five types of sample size and power calculations as listed below. These five types can be adapted in various ways to encompass many of the standard designs encountered in practice.

Click on the design to open the computational routine. Click on 'example' to see explanations and examples of each type, drawn from Lee (2004).

Sample Size Calculations for
a. Completely randomized treatment-control designs (example)
b. Matched-pairs designs (i.e. randomized block designs) (example)

Power Calculations for
a. Completely randomized treatment-control designs (example)
b. Matched-pairs designs (i.e. randomized block designs) (example)
c. Multiple-treatment designs having an isolated treatment effect (example)

\section{Cited References}

1. Lee, M.-L. T. (2004). Analysis of Microarray Gene Expression Data, Kluwer Academic Publishers, ISBN 0-7923-7087-2.

2. Lee, M.-L. T., Whitmore, G. A. (2002). Power and sample size for DNA microarray studies, Statistics in Medicine, 21:3543-3570

\section{Acknowledgements}

This project was support by NIH grant HG02510 (Lee, M-L.T.). The computer routines supporting this website have been developed and are maintained by Dr. Weiliang Qiu, Postdoctoral Research Fellow, Channing Laboratory, Brigham and Women's Hospital and Harvard Medical School, Boston.

Last Modified: 01/25/2006 11:02:06

http://www.biostat.harvard.edu/people/faculty/mltlee/web-front-r.html2006-1 1-5 21:02:46

Figure 1: A snapshot of the home webpage of SPCalc any medium, for non-commercial purposes, provided the original author and source are credited. 